\title{
Analysis and Interpretation of Electromagnetic Induction data in the Frequency Domain (FDEM) and Magnetic data applied to hydrogeology, in the Samambaia-PE region.
}

Marcos César Soares De Queiroz *1, Mônica Giannoccaro Von Huelsen ${ }^{2}$ and Gustavo Lopes Costa 2

\author{
Copyright 2021, SBGf - Sociedade Brasileira de Geofísica. \\ This paper was prepared for presentation during the $17^{\text {th }}$ International Congress of the Brazilian Geophysical Society held in Rio de Janeiro, Brazil, 8-11 November 2021 \\ Contents of this paper were reviewed by the Technical Committee of the $17^{\text {th }}$ International Congress of the Brazilian Geophysical Society and do not necessarily represent any position of \\ the SBGf, its officers or members. Electronic reproduction or storage of any part of this paper for commercial purposes without the written consent of the Brazilian Geophysical Society is \\ prohibited.
}

The Northeast region of Brazil presents a crystalline basement composed of granite-gneiss-migmatitic rocks. The dominant climate is semi-arid and characterized by low and irregular rainfall rates, in addition to high rates of insolation and evaporation, which disadvantages the retention of water for long periods in shallow reservoirs. Is the type of terrain, percolation and accumulation of groundwater area predominantly controlled by fractures. Within this context, high resolution airborne geophysical data were analyzed, and their generated products were integrated with available geological and hydrogeological data.

From the electromagnetic signal, images of the Quadrature (Q) and in-phase (I) componentes in Frequency Domain (FDEM) were obtained, with three pairs of coplanar horizontal coils and two pairs of coaxial vertical coils, spaced $6.4 \mathrm{~m}$ apart. The coaxial coils are in the vertical position, with frequencies of 900 and $4500 \mathrm{~Hz}$ and the axis aligned with the direction off light, and the coplanar coils are horizontal, with frequencies of 900,4500 and $33.000 \mathrm{~Hz}$. Stacked conductivity profiles were generated for diferente frequencies, low, médium and high, considering the coaxial and coplanar arrangements. From the image of the Analytic Signal Amplitude (ASA), where it is noted that the region with the highest magnetic values predominates in the southeast of the area. In general, the magnetic structures have a preferred NE / SW direction. According to the power spectrum of the magnetic signal the deeper anomalous sources reach values below $2 \mathrm{~km}$. The shallow sources are $0.5 \mathrm{~km}$ deep. The image of the apparent conductivity shows high conductivity in the northwest direction, with predominance structures in the NE / SW practically in the whole area. This extensive conductive area to NW was interpreted as a probable conductive cover due to the existing rocks in the region or even may be related to the presence of groundwater that is in that part. The stacked profiles indicate shallow southeast structures characterized with high frequency electromagnetic signal in NW / SE directions and an NE / SW. Most of the electromagnetic structures do not present displacement with the increase of the depth, indicating that these structures are arranged vertical to subvertical. It is evident that the sinistral NE / SW transcurrent faults and N / S extension, which cuts the area, caused the displacement of the electromagnetic anomaly. Depth conductivity imaging (CDI) was integrated with information from tubular wells registered in SIAGAS/CPRM, thus discriminating regions characterized by groundwater accumulation, where the conductive behavior of the medium presented high gradients. Conductor geometry was interpreted using conductivity profiles indicating a high presence of subvertical and vertical fractures. With respect to magnetometry, the depths of the magnetic sources were estimated using the radial power spectrum and the euler deconvolution. Euler's solutions were integrated with the CDI's images, the geological model and the hydrogeological information for joint interpretation, indicating magnetic structures associated with high conductive gradients, which can characterize areas of hydrogeological interest. Tubular wells with higher flow are found in a high conductive gradient and with the presence of subverticalized magnetic structures. 